\title{
CONF-9509238--1
}

UCRL-JC-120994

PREPRINT

\section{Effect of ELMs on the SOL Plasma in DIII-D}

\section{R.A. Jong, G.D. Porter, R.J. Groebner, C.J. Lasnier, GA Thomson Team}

This paper was prepared for submittal to the

IAEA Technical Committee Meeting/U.S. Japan Workshop on H-Mode Physics

Princeton, New Jersey

September 18-20, 1995

May 9, 1995

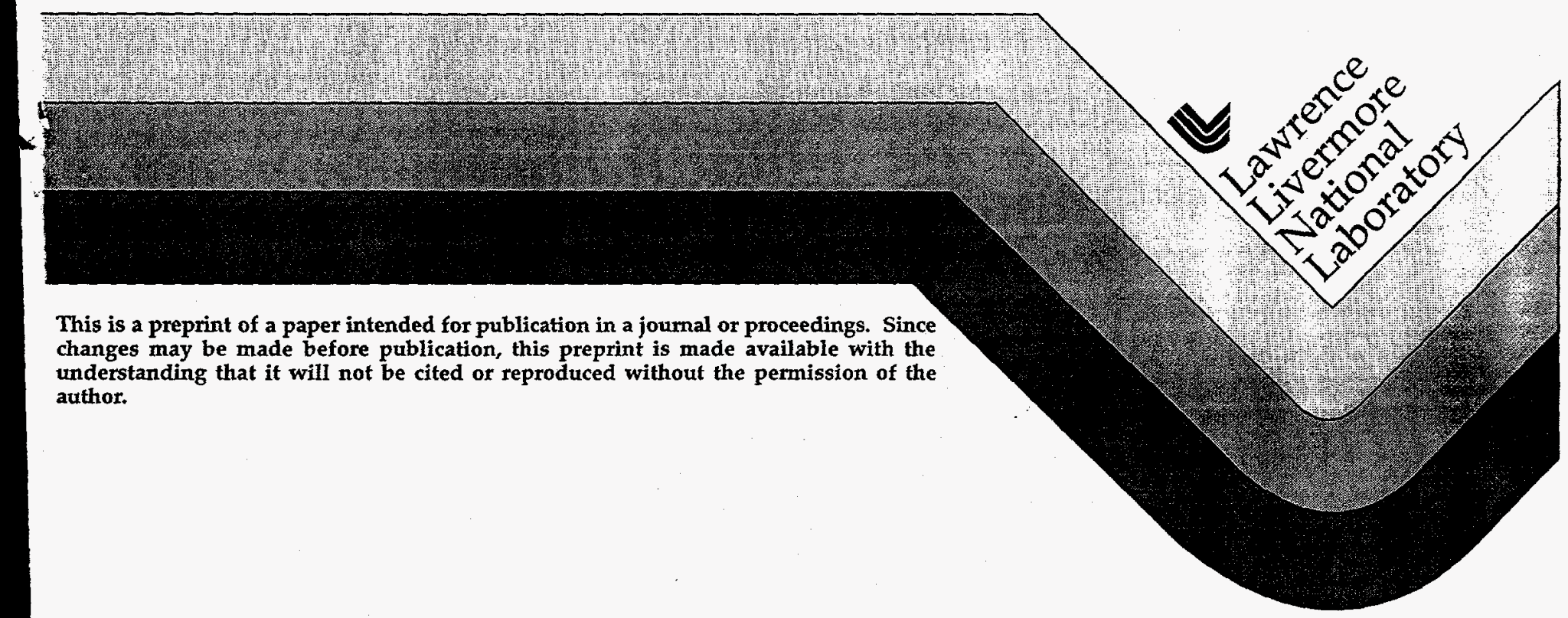

\section{MASTER}




\section{DISCLAIMER}

This document was prepared as an account of work sponsored by an agency of the United States Government. Neither the United States Government nor the University of California nor any of their employees, makes any warranty, express or implied, or assumes any legal liability or responsibility for the accuracy, completeness, or usefulness of any information, apparatus, product, or process disclosed, or represents that its use would not infringe privately owned rights. Reference herein to any specific commercial product, process, or service by trade name, trademark, manufacturer, or otherwise, does not necessarily constitute or imply its endorsement, recommendation, or favoring by the United States Government or the University of California. The views and opinions of authors expressed herein do not necessarily state or reflect those of the United States Government or the University of California, and shall not be used for advertising or product endorsement purposes. 


\section{DISCLAIMER}

Portions of this document may be illegible in electronic image products. Images are produced from the best available original document. 


\title{
Effect of ELMs on the SOL Plasma in DIII-D
}

\author{
R.A. Jong, G.D. Porter, R.J. Groebner, C.J. Lasnier, GA Thomson Team \\ Lawrence Livermore National Laboratory \\ P.O. Box 808 \\ Livermore, California 94551 \\ General Atomics \\ 3550 General Atomics Court \\ San Diego, California 92121
}

Classification Number : 5225 Plasma Properties

Prepared for the IAEA Technical Committee Meeting on

H-Mode Physics / U.S. - Japan Workshop on H-Mode Physics

5th Workshop on H-Mode Physics

at

Princeton Plasma Physics Laboratory

Princeton University

James Forrestal Campus

Princeton, New Jersey

18 - 20 September 1995

and to be published in the Proceedings in Plasma Physics and Controlled Fusion 


\title{
Effect of ELMs on the SOL plasma in DIII-D
}

\author{
R.A. Jong ${ }^{\dagger}$, G.D. Porter ${ }^{\dagger}$, R.J. Groebner ${ }^{\ddagger}$, C.J. Lasnier ${ }^{\dagger}$ and GA Thomson \\ Team $\ddagger$ \\ $\dagger$ Lawrence Livermore National Laboratory, Livermore CA 94550, USA \\ $\ddagger$ General Atomics, San Diego CA 92121, USA
}

\begin{abstract}
We have studied the evolution of the edge plasma in VH-mode discharges in DIII-D as the discharge evolves from the ELM-free H-mode phase through the VH-mode phase to the final ELMing $\mathrm{H}$-mode phase, by following the changes in the radial profiles of the density and temperature, in the core plasma near the separatrix and in the scrape-off-layer (SOL) plasma outside the separatrix. The electron density and temperature profiles in the SOL do not show any significant difference between the ELM-free $\mathrm{H}$-mode and VH-mode phases. In the ELMing phase, the density profile broadens during an ELM, forming a high density $\left(\mathrm{n}_{\mathrm{e}}>1 \times 10^{19} / \mathrm{m}^{3}\right)$ plateau that extends out into the SOL to the limit of the measurement. This density plateau persists between the ELMs, although the density in the SOL does relax somewhat between the ELMs, with a characteristic time that can be larger than ten milliseconds, much longer than the sonic particle flow time to the divertor plates. The time average density scale length measured at the separatrix increases by about a factor of two after the ELMs begin. This density scale length increases with the ELM background, as measured by the photo-diodes nearest to, but outside the outer strike-point, suggesting that recycling from the divertor targets is supplying particles that maintain the density plateau. The electron temperature profile in the SOL also broadens during an ELM, but relaxes on a much faster time scale and does not persist between ELMs.
\end{abstract}

\section{Introduction}

The scrape-off-layer (SOL) plasma in tokamak discharges has been the subject of several recent studies on DIII-D by Watkins et al 1995, Watkins et al 1994, Jong et al, Lippmann $e t a l$, and Schaffer $e t a l$. In addition, Hill et al studied the effect of ELMs on edge plasma scaling in DIII-D. The density and temperature profiles in the edge region of the plasma and in the divertor region have obvious importance in the study of neutral fueling of the core plasma, impurity penetration of the core plasma, and the transport of particles and energy from the core plasma to the SOL and divertor regions. Here, we shall describe experiments that followed the evolution of the plasma in the scrape-off layer and divertor regions during the quiescent $\mathrm{H}$-mode, VH-mode, and ELMing $\mathrm{H}$-mode phases of high current (1.6 MA), high triangularity, double-null discharges in the DIII-D tokamak.

We have measured the edge and divertor plasmas with a wide array of diagnostics including Thomson scattering for the electron density and temperature, charge exchange recombination (CER) for the ion temperature, a reciprocating probe for midplane electron density and temperature profiles, infrared TV (IRTV) cameras for heat flux distributions on the divertor floor, Langmuir probes for electron temperature and density on the divertor floor, and photo-diodes to measure the $\mathrm{D}_{\alpha}$ light in the divertor. Because of space limitations here, we shall concentrate most of our discussion on the measurements from the multi-pulse 
Thomson scattering diagnostic to follow the changes in the radial profiles of the electron density and temperature. We shall also briefly discuss the heat flux profiles measured with the IRTV diagnostic looking at the divertor floor. The whole array of diagnostics are consistent with edge electron density and temperature profiles in the SOL and heat flux profiles in the divertor that do not show any significant difference between the ELM-free Hmode and VH-mode phases. We find that the profiles are significantly broader during an ELM, and that the broad density profiles can persist in the time between the ELMs.

\section{Typical Long-Duration VH-Mode Discharge}

The typical high current, high triangularity, double-null discharge we consider is shot number 83618. This is a VH-mode plasma with a long ELM-free period of almost $900 \mathrm{ms,}$ extending from about 2000 to $2877 \mathrm{~ms}$. As seen in figure 1, the ELM-free H-mode transitions to a VH-mode around $2400 \mathrm{~ms}$ (i.e. $\tau_{\mathrm{th}} / \tau_{\mathrm{DIII}-\mathrm{D} / \mathrm{JET}} \geq 1.6$ ). This VH phase lasts for about $500 \mathrm{~ms}$, with an energy confinement time enhancement value of up to 2.5 comparedto the DIII-D/JET H-mode scaling). The end of the VH-mode phase is signaled by the event at $2877 \mathrm{~ms}$ where the photo-diode signal increases and indicates the onset of ELMs. At the same time, there is a large drop in the plasma pressure as indicated by the drop in the toroidal $\beta$ (BETAT).

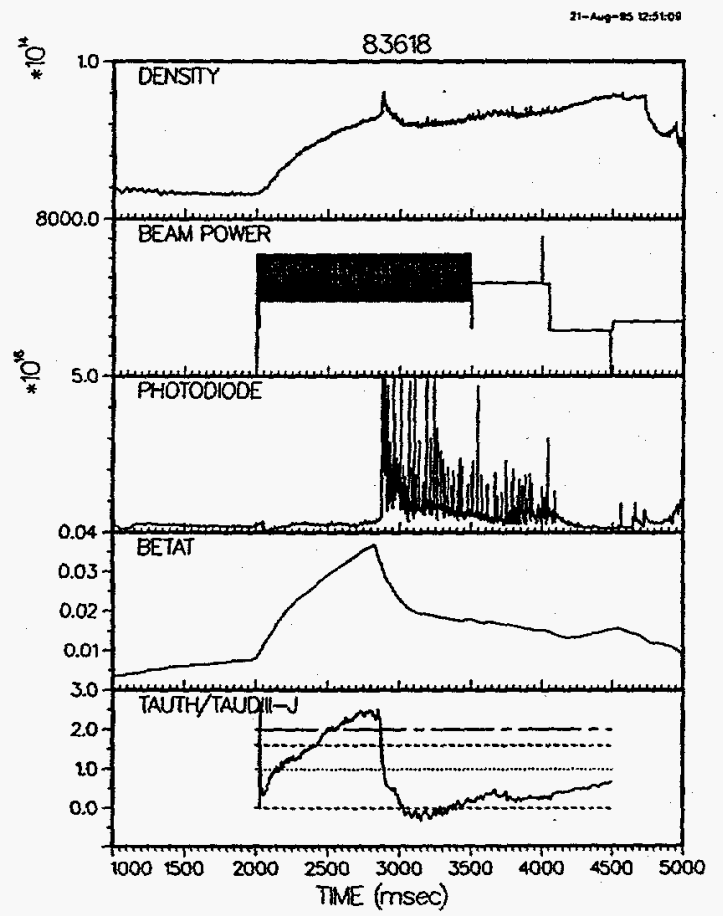

Figure 1: Time history

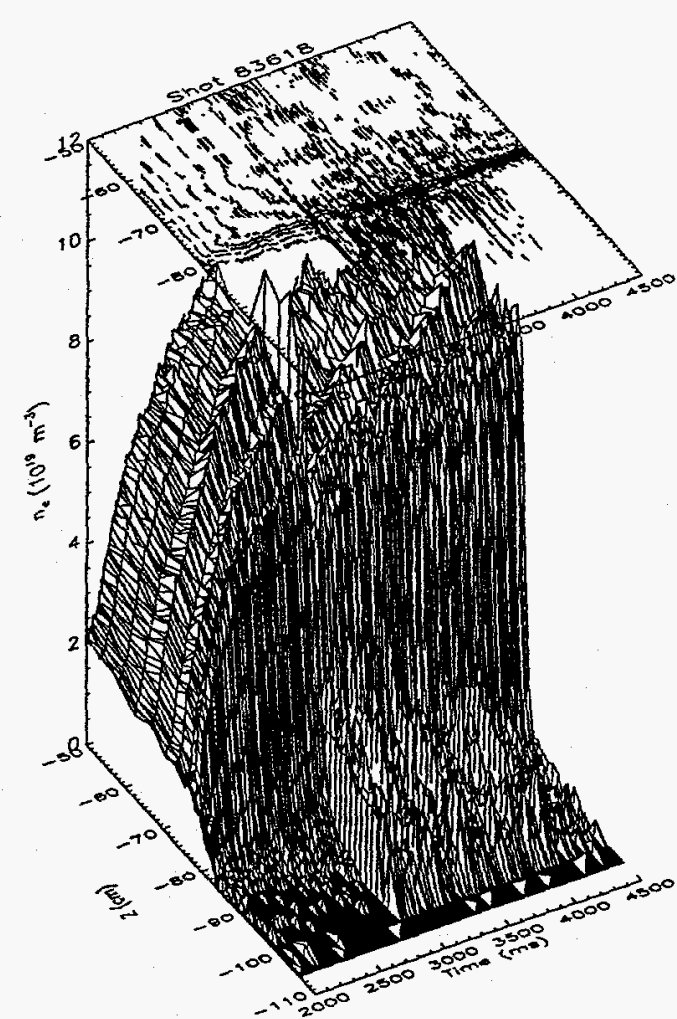

Figure 2 : Electron density contours

The 2-D and 3-D electron density contours measured by the multi-pulse Thomson diagnostic are shown in figure 2 . There is a dramatic change in the contours at the end of the VH-mode. A density plateau extending out to the limit of the measurement is clearly observed after the onset of ELMs and persists to the end of the discharge In contrast, during the ELM-free period there is no significant density outside the separatrix (around $Z=81 \mathrm{~cm}$ ), and the density is not measurable by the Thomson scattering diagnostic beyond the $90 \mathrm{~cm}$ elevation.

The electron density profiles can also be displayed as a function of the distance measured from the separatrix, when mapped to the plasma midplane. We can see the differences in the 
profiles during the ELM-free $\mathrm{H}$-mode, $\mathrm{VH}$-mode and ELMing $\mathrm{H}$-mode phases in figure 3. At the 2300 and $2875 \mathrm{~ms}$ times during the ELM-free $\mathrm{H}$-mode and VH-mode phases respectively, the SOL density profiles are nearly exponential, with insignificant density outside the separatrix in the SOL. After the onset of the ELMs, the density profile broadens during an ELM and forms a high density plateau extending well into the SOL, with $n_{e}>1 \times$ $10^{19} / \mathrm{m}^{3}$. The density value can persist between the ELMs, often for many laser pulses, which in this case are $6 \mathrm{~ms}$ apart.
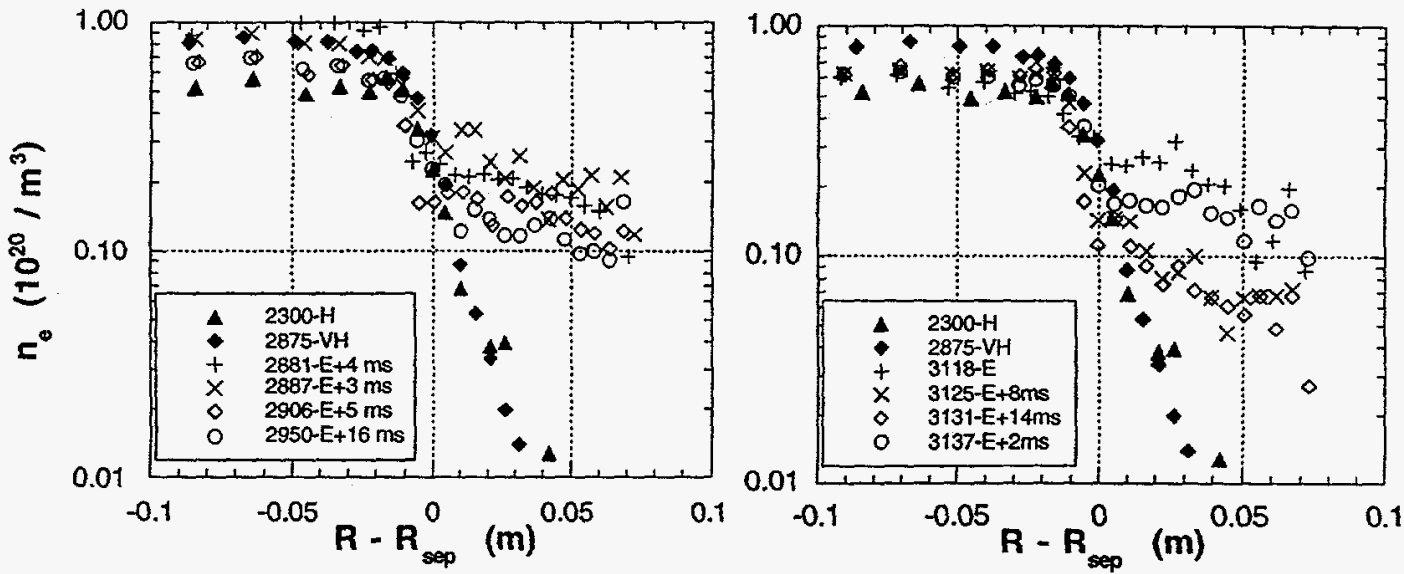

Figure 3 : Electron density profiles in ELM-free $\mathrm{H}$-mode $(2300 \mathrm{~ms})$ $\mathrm{VH}-$ mode $(2875 \mathrm{~ms})$ and at various times between the ELMs

In the time period immediately after the end of the VH-mode (2881-2950 ms), the ELMs are rapid and large. The measured density plateau values remain above the $1 \times 10^{19} / \mathrm{m}^{3}$ level and there is very little density profile relaxation in this period. Later in the ELMing Hmode phase when the ELMs are smaller and more isolated, we can see the density profile relax between the ELMs. In figure 3, we also show the density profile variation after an ELM at $3118 \mathrm{~ms}$. The profiles for the time at $3118 \mathrm{~ms}$ (during the ELM) as well as for the later profiles at 3125,3131 , and $3137 \mathrm{~ms}$ demonstrate the profile relaxation between ELMs. The times 3125 and 3131 are $7 \mathrm{~ms}$ and $14 \mathrm{~ms}$ after the peak of the ELM at $3118 \mathrm{~ms}$, and the density values for these profiles have dropped by about a factor of 3 relative to the value at $3118 \mathrm{~ms}$. The $3137 \mathrm{~ms}$ profile is $2 \mathrm{~ms}$ after the peak of the next ELM, and the density value has decreased only slightly from the 3118 value. See figure 4 for a plot of the laser pulse times relative to the ELMs shown in the photo-diode signal. While the density relaxes more rapidly between these smaller, less frequent ELMs, the profile in the SOL still does not revert to it's narrower, pre-ELM shape.

The temporal variation in the SOL thickness is clearly shown by the plot of the density scale length in figure 5 . The density scale length mapped to the midplane is defined in terms of the separatrix values of density and density gradient as

$$
\mathrm{L}_{\mathrm{n}}=\mathrm{n}_{\mathrm{e}} /\left(\mathrm{dn} \mathrm{n}_{\mathrm{e}} / \mathrm{dr}\right)
$$

The scale length is plotted versus time in figure 5 along with the ELM background signal from the upper photo-diodes closest to, but outside the outer divertor strike points. Here, the ELM background is obtained by a linear fit to the photo-diode signal over a span of $100 \mathrm{~ms}$, with the excursions due to the ELMs removed.

The time average density scale length appears to correlate with the background photodiode signals near the outer strike point. Because of the $100 \mathrm{~ms}$ averaging time used in the algorithm for the ELM analysis, the ELM background signal from the upper photo-diode, 
PHD02UP, in figure 5 is effectively shifted to lairger times by up to $100 \mathrm{~ms}$. However, we can see that during most of the ELM-free $\mathrm{H}$-mode period from 2200 to $2400 \mathrm{~ms}$, and the VH-mode phase from 2400 to $2875 \mathrm{~ms}$, the density scale length is constant at around 0.008

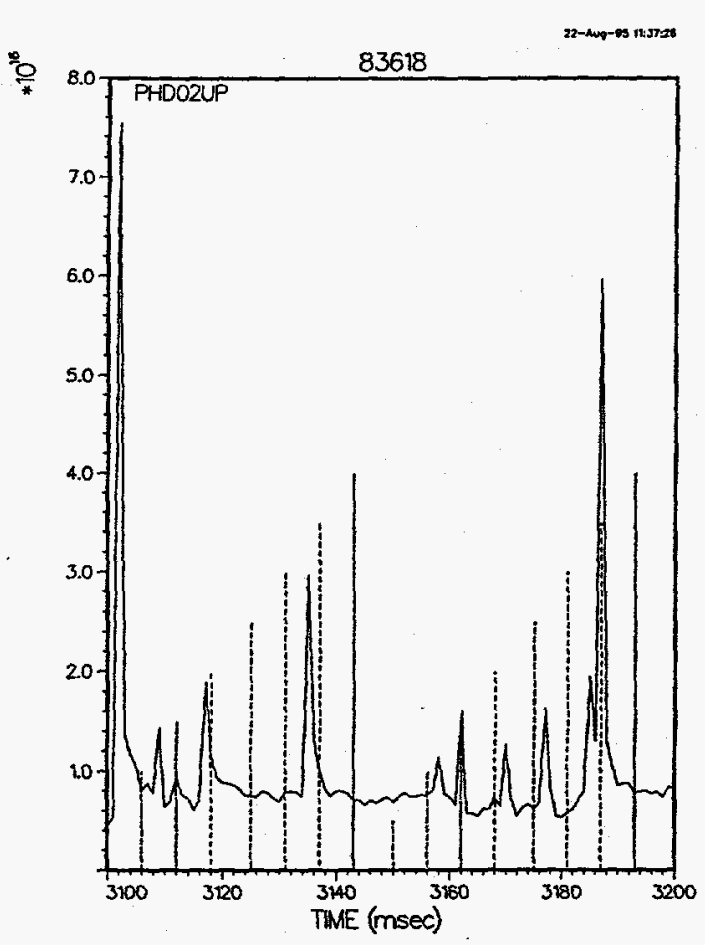

Figure 4 :Photo-diode signal and Thomson laser pulse times for ELMing period around $3118 \mathrm{~ms}$. $\mathrm{m}$ and the photo-diode signal is correspondingly low.

When the ELM activity starts after 2877 $\mathrm{ms}$, the density profiles are modulated by the ELM activity. This modulation, in conjunction with the uncontrolled time of the laser pulse relative to the time of occurrence of the ELM, can produce the apparent scatter in the scale length, $L_{n}$, seen after $2900 \mathrm{~ms}$ in figure 5 . We are measuring the density at times during an ELM and also at various times during the density relaxation phase between ELMs, so the density scale length can change from laser pulse to laser pulse. However, the average value increases to about $0.013 \mathrm{~m}$ in the time span from 2900 to $3500 \mathrm{~ms}$, concurrent with the increase in the photo-diode signals. In the period from 3500 to $3800 \mathrm{~ms}$, the background photo-diode signal and the average scale length decrease together.

In the ELMing $\mathrm{H}$-mode phase, the average value of the density scale length increases by about $65 \%$ over the $\mathrm{VH}$-mode and ELM-free $\mathrm{H}$-mode value. The correlation of the density scale length with the photo-diode background signal suggests that the recycling from the divertor is supplying particles to support the density

plateau in the SOL. The ELMs produce a transient plasma in the SOL which supplies the power to ionize the recycled particles to sustain the broad density profiles between the ELMs.

The corresponding scale length of the electron temperature (not shown here), also increases during an ELM. Because of the rapid conduction of the electron power to the divertor on the open field lines and the absence of a

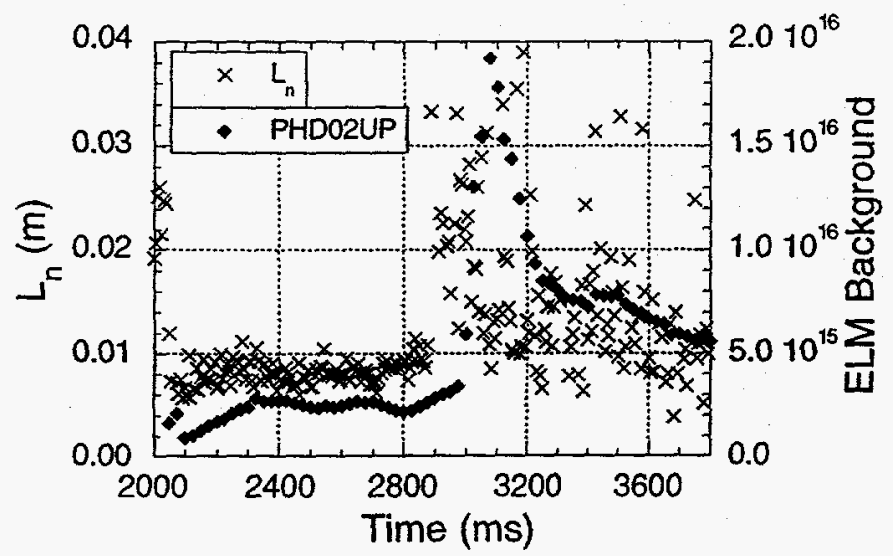

Figure 5: Density scale length and background photodiode signal for shot 83618 . VH-mode extends from $\sim 2400$ to $2877 \mathrm{~ms}$. power source in the divertor for the electrons, the relaxation of the $T_{e}$ scale length to its quiescent phase value is much quicker than for the density scale length. Hence, while the average value of the $T_{e}$ scale length we calculate during the ELMing $\mathrm{H}$-mode phase is larger, the lower bound of the scale length is nearly the same as is was in the ELM-free period. Overall, the $\mathrm{T}_{\mathrm{e}}$ scale length is about one half of the density scale

length. 
The measurement of the divertor heat flux profile for the shot discussed here lacks the time resolution necessary to determine the heat flux profile between the ELMs. What we effectively measure is the heat flux over a time span that includes several ELMs. Experiments with data taken at a higher sampling rate to give the behavior between ELMs are scheduled for a later time. However, the data does show that the maximum in the peak power and total power to the divertor both occur at the end of the VH-mode. The peak power and total power remain at elevated values until shortly after about $3000 \mathrm{~ms}$, at which time the VH-mode ending event is over, and a normal ELMing H-mode discharge follows.

During the quiescent phases of the discharge, the FWHM of the outer power peak is about $10 \mathrm{~cm}$ and very slowly increasing. When the ELMs begin, the width almost doubles for a period of less than $100 \mathrm{~ms}$, and then drops back to a level above the pre-event value. The width continues to increase, until later in the ELMing $\mathrm{H}$-mode phase, it is again about double its value in the $\mathrm{VH}$-mode and ELM-free $\mathrm{H}$-mode phases, consistent with a wider SOL plasma during the ELMing plasma phase.

\section{Summary}

The vast array of diagnostics that view the SOL and divertor plasmas in the DIII-D tokamak indicate that the SOL plasma density is nearly exponential in the quiescent $\mathrm{H}$-mode and $\mathrm{VH}-$ mode phases, and becomes broader by forming a high density plateau during ELM activity in high current, high triangularity, double-null plasmas. The broad SOL density profile persists between the ELMs, albeit with some density relaxation to lower values. The source of particles maintaining the broad density profile appears to be recycling from the divertor floor as indicated by the correlation of the SOL density with the $\mathrm{D}_{\alpha}$ light at the divertor strike points. Unlike the case of the density, where recycling or gas feed can support a broad density plateau in the SOL in the presence of the ELMs, there is no source of power to maintain a broad electron temperature profile in the SOL during the period between the ELMs. Consequently, the electron temperature profile which also broadens during an ELM, does not persist between the ELMs. The heat flux and density profiles measured on the divertor floor are also broader after the appearance of ELMs when compared to the VHmode and ELM-free $\mathrm{H}$-mode profiles.

\section{Acknowledgments}

This work was performed under the auspices of the U.S. DOE by Lawrence Livermore National Laboratory under Contract No. W-7405-ENG-48 and by General Atomics under Contract. No. DE-AC03-89ER51114.

\section{References}

Hill D N et al 1992 J. Nucl Mat. 204-209 204-209.

Jong R A, Casper T A, Lasnier C J 1994 Bull. Am. Phys. Soc. 391651.

Lippmann S I, Evans T E, Jackson G L, West W P 1992 J. Nucl Mat. 196-198 498.

Schaffer M J et al (1993) Plasma Physics and Controlled Nuclear Fusion Research 1992 (Proc. 14th Int. Conf., Würzburg) Vol. 1 (IAEA, Vienna) 299.

Watkins J G, Moyer R A, Jong R A, LaHaye R J, Evans T E, Hill D N, Stambaugh R D 1994 Bull. Am. Phys. Soc., 391653.

Watkins J G et al 1995 J. Nucl Mat. 220-222 347. 